\title{
Fístula postmanga gástrica laparoscópica
}

\author{
Fistula after laparoscopic sleeve gastrectomy \\ César Romero Mejía, * Omar Paipilla Monroy*
}

Palabras clave: Manga gástrica laparoscópica, fuga postmanga, fístula tardía postmanga laparoscópica.

Key words: Laparoscopic sleeve gastrectomy, leaks after sleeve gastrectomy, late fistula after sleeve gastrectomy.

* Servicio de Cirugía General, Hospital General de Tijuana, Baja California.

Recibido: 07/09/2016 Aceptado: 16/12/2016

\section{RESUMEN}

\section{ABSTRACT}

Se presentan cuatro pacientes con fuga gástrica tras gastrectomía en manga por laparoscopia para el tratamiento de obesidad mórbida. Los individuos operados en otro hospital ingresaron, en promedio, al décimo día postoperatorio. El sitio de fuga fue por abajo de la unión gastroesofágica. El tamaño de la perforación fue, en promedio, de dos centímetros. A los sujetos se les realizaron cuatro intervenciones quirúrgicas, con estancia hospitalaria de 20 días. Tres personas se recuperaron y una solicitó su envío a otro hospital en la Ciudad de México. Se comenta la clasificación clínico-terapéutica del manejo de estas lesiones en tres categorías: tempranas, intermedias y tardías. Todos fueron catalogados como formas tardías. Los mecanismos aparentes de lesión fueron daño térmico, creación de un tubo de alta presión adyacente a dos esfínteres y el uso empírico de grapas de diferente medida. Finalmente, se analizan las nuevas formas para el tratamiento de las fugas gástricas tardías postmanga laparoscópica.
We present four patients with gastric leak after sleeve gastrectomy for the treatment of morbid obesity. All of them came from another hospital and were admitted an average of 10 days after surgery. The perforation was located at the esophagogastric junction and measured two centimeters. Every patient was operated upon four times, with a hospital stay of 20 days. Three patients closed the fistula and one requested his transfer to another hospital in Mexico City. We review a clinical-therapeutic classification for the management of these lesions and attempt to explain the mechanisms of production and the way to treat them. Lesions are classified in three categories: early, intermediate and late. All of our patients had late leaks. The nature of the lesion was described as thermal tissue damage, the creation of a high-pressure tube adjacent to two sphincters and the empirical use of different sizes of staples. Finally, we review the new forms of treatment for late post sleeve gastrectomy leaks.

\section{INTRODUCCIÓN}

$\mathrm{E}^{\mathrm{n}}$ n México ha aumentado la obesidad extrema y su tratamiento. Los pobres resultados de algunos procedimientos restrictivos, además del costo de insumos en engrapadoras quirúrgicas, han hecho que se intenten nuevas formas de abordaje.

En un inicio, la manga gástrica por laparoscopia (MGL) fue propuesta como el primer paso quirúrgico en pacientes de alto riesgo con índice de masa corporal mayor a 65 que se someterían a derivación gástrica. Aunque los individuos no llegaron a pesos ideales, se observó que disminuyeron de peso y se redujeron las comorbilidades, por lo que el interés de los cirujanos por ofrecer un método seguro y con resultados a largo plazo sin mutilar el tubo digestivo ni alterar la absorción de nutrientes hizo que esta cirugía se ofreciera como alternativa en sujetos con obesidad mórbida. ${ }^{1}$
Sin embargo, series grandes mostraron que la MGL no está exenta de complicaciones; una forma grave es la fístula gástrica por dehiscencia de la línea de engrapado. ${ }^{2}$ El objetivo del presente trabajo es mostrar cuatro casos de fístula gástrica después de MGL, su estudio, tratamiento y evolución; así como usar una clasificación clínico-terapéutica para esta catástrofe quirúrgica y comentar las nuevas teorías de su génesis y los métodos más novedosos de manejo.

\section{REPORTE DE CASOS}

Se describen cuatro sujetos operados de manga gástrica por laparoscopia en otros hospitales y referidos al Hospital General de Tijuana para su atención. Todos tenían fístula gástrica en la cara posterior del ángulo de Hiss.

El cuadro / muestra las características de las personas, edad, sexo, sitio y tamaño de la 
fuga, los procedimientos quirúrgicos realizados en nuestro hospital, los días de estancia hospitalaria y el resultado final.

Se trató a cuatro pacientes, tres mujeres y un hombre, con edad de 30 a 40 años, operados de cinco a 21 días antes. Fueron reintervenidos con cirugía abierta y se realizó lavado quirúrgico, sutura en el sitio de la fístula con monofilamento Prolene ${ }^{\circledR}$ 00, aplicación de goma quirúrgica Tissucol ${ }^{\circledR}$ y drenaje cerrado tipo Blake. En tres individuos falló el intento de tratamiento con endoprótesis colocada en nuestro hospital; en todos los casos, el sitio de fístula fue posterior y en la unión esofagogástrica; el tamaño varió de uno a cuatro centímetros, y se acompañó de absceso subfrénico izquierdo con periesplenitis, que fue resuelta con esplenectomía. Todos recibieron apoyo nutricional, soluciones parenterales y triple esquema de antibióticos (cefotaxima, amikacina y metronidazol). En todos se colocó sonda nasogástrica multiperforada para disminuir la presión en el esófago y manga gástrica.

\section{RESULTADOS}

Los cuatro sujetos sobrevivieron y egresaron del hospital. En tres se logró el cierre de la fístula en nuestra institución y una persona fue trasladada a otro hospital, donde se recolocó una endoprótesis que permaneció por un año. El cuadro I muestra los resultados de los cuatro pacientes. La permanencia hospitalaria fue de 10 a 35 días, con un promedio de 20. El número de cirugías por individuo fue de cuatro. Todos fueron sometidos a lavado y drenaje en quirófano en forma programada dos a tres veces por semana hasta el control de la fístula.

\section{DISCUSIÓN}

Los estudios de Yehoshua muestran que la presión en el esfínter esofágico inferior disminuye en la MGL; sin embargo, la presión intragástrica aumenta a $43 \pm 8 \mathrm{mmHg}$, contra $34 \pm 6$ $\mathrm{mmHg}$ en un estómago promedio, justificada por falta de distensibilidad del cuerpo y fundus

\section{Cuadro I. Pacientes.}

\begin{tabular}{|c|c|c|c|c|c|c|c|}
\hline Paciente & Edad & Sexo & $\begin{array}{l}\text { Días de } \\
\text { cirugía } \\
\text { inicial }\end{array}$ & $\begin{array}{c}\text { Sitio de } \\
\text { fuga }\end{array}$ & Procedimiento & DEH & Resultado \\
\hline 1 & 36 & $\mathrm{~F}$ & 7 días & $\begin{array}{c}1 \mathrm{~cm} \\
<\text { Hiss }\end{array}$ & $\begin{array}{l}\text { LDQ } \\
\text { Sutura quirúrgica, } \\
\text { esplenectomía }\end{array}$ & 10 días & Alta con cierre \\
\hline 2 & 40 & M & 21 & $\begin{array}{c}4 \mathrm{~cm} \\
<\text { Hiss }\end{array}$ & $\begin{array}{l}5 \text { LDQ } \\
\text { Sutura, goma Qx, } \\
\text { esplenectomía }\end{array}$ & 35 & Alta con cierre \\
\hline 3 & 36 & $\mathrm{~F}$ & 7 & $\begin{array}{l}2.5 \mathrm{~cm} \\
<\text { Hiss }\end{array}$ & $\begin{array}{l}3 \text { LDQ } \\
\text { Sutura, goma Qx, } \\
\text { esplenectomía, } \\
\text { endoprótesis fallida }\end{array}$ & 21 & Alta con cierre \\
\hline 4 & 30 & $\mathrm{~F}$ & 5 & $\begin{array}{l}2.5 \mathrm{~cm} \\
<\text { Hiss }\end{array}$ & $\begin{array}{l}3 \text { LDQ } \\
\text { Sutura, goma Qx, } \\
\text { esplenectomía. } \\
\text { SNG/MO } 2 \text { días CPAP, } \\
\text { endoprótesis fallida }\end{array}$ & 16 & $\begin{array}{l}\text { Traslado } \\
\text { a tercer nivel; } \\
\text { endoprótesis } \\
\text { por un año }\end{array}$ \\
\hline$\sim$ & 38 & & 10 & $2 \mathrm{~cm}$ & & 20 & \\
\hline
\end{tabular}

$\mathrm{LDQ}=$ Lavado y drenaje quirúrgico; $\mathrm{SNG} / \mathrm{MO}$ = Sonda nasogástrica y medicamentos orales; $\mathrm{DEH}=$ Días de estancia hospitalaria; = Promedio; $\mathrm{Qx}=$ Quirúrgica; $\mathrm{CPAP}=$ Presión positiva continua en vía aérea. 


Cuadro II. Clasificación de fugas post MGL.
\begin{tabular}{|lll} 
Tempranas & Intermedias & \multicolumn{1}{c}{ Tardías } \\
\hline $\begin{array}{ll}\text { De 1 a 3 días } \\
\text { PO }\end{array}$ & $\begin{array}{l}\text { De 4 a } 7 \text { días } \\
\text { PO }\end{array}$ & $\begin{array}{l}\text { de } 8 \text { días } \\
\text { PO }\end{array}$ \\
\hline
\end{tabular}

Burgos AM, Braghetto I, Cscendes A, Maluenda F, Korn O, Yarmuch J, et al. Gastric leak after laparoscopicsleeve gastrectomy for obesity. Obes Surg. 2009; 19: $1672-1677.5$

gástrico. ${ }^{3}$ La creación de un tubo de alta presión adyacente a dos esfínteres, cardias y píloro, crea un estómago reducido y sin posibilidad de distensión, con una bomba antral y dos válvulas adyacentes, y eleva la presión en la luz gástrica. Además, la sección de los ligamentos gastrofrénico y gastroesplénico por arriba de los vasos cortos se asocia frecuentemente a daño térmico por uso del bisturí armónico en esta área, lo que explica que las fugas más frecuentes son posteriores y por abajo de la unión esofagogástrica.

Las fístulas post-MGL son poco frecuentes, según Menenakos en un estudio de 261 sujetos seguidos a un año y con incidencia de 3.8\% (10 casos). A ocho personas se les colocó endoprótesis, con curación en cinco; dos requirieron gastrectomía total y uno persistió con fístula crónica. ${ }^{4}$ De los dos restantes, uno falleció al arribo y otro cerró en forma espontánea con curaciones y nutrición parenteral total.

En la Universidad de Chile clasifican las fístulas posteriores a MGL de acuerdo con el tiempo de su presentación, en tempranas (de uno a tres días), intermedias, (de cuatro a siete días) y tardías (> ocho días) (Cuadro II). ${ }^{5}$ Lo común es que las fugas tempranas sean más fáciles de tratar, con menor mortalidad.

Se ha descrito el uso de endoprótesis cubiertas con silicón para el manejo de fístulas post-MGL. Serra y su grupo comunicaron seis pacientes con endoprótesis recubiertas autoexpandibles, con control en cinco individuos. ${ }^{6}$ Kauer y sus colegas usaron endoprótesis recubiertas con silicón y obtuvieron el control definitivo de nueve de 10 sujetos con fístula post-MGL. ${ }^{7}$ Sin embargo, otros señalan resultados adversos, como migración y persistencia de la fístula, al usar endoprótesis. ${ }^{8}$ Lindenmann en Austria, usa endoprótesis metálicas traqueales cubiertas para tratar fístulas y muestra migración en dos de seis casos. ${ }^{9}$

Ramos y su equipo describen ocho personas sometidas a gastrectomía total postfístula por MGL, con edad promedio de 46 años, tres intentos de cierre y duración de un año; recomiendan el tratamiento agresivo si la fístula persiste por más de seis meses y consideran que éstas son más difíciles de tratar que las que aparecen tras una derivación gástrica. ${ }^{10}$

En un estudio hecho por Huerta y colaboradores en 1,067 pacientes operados de derivación gástrica en quienes se utilizó aparato de presión positiva continua en vía aérea (CPAP), en 490 de ellos con apnea de sueño y 159 dependiente de CPAP, sólo hubo 15 fugas, dos de ellas en individuos dependientes, pero no es recomendable utilizarlo en $M G L$, pues su seguridad no se ha establecido aún. ${ }^{11}$

Otras medidas preventivas son el uso de bujía como guía no menor a 34 fr y la inyección de toxina botulínica en píloro durante la intervención para prevenir la fuga. ${ }^{12}$

La presión intraluminal y el vaciamiento gástrico en la MGL están aumentados, por lo que el riesgo de fuga es mayor comparado con la derivación gástrica por laparoscopia. ${ }^{13}$ Sugerimos que ante la sospecha de fuga, los sujetos sean intervenidos en forma temprana; si el diagnóstico es tardío, las exploraciones en forma programada son superiores a las de libre demanda, además de nutrir y drenar adecuadamente a la persona. ${ }^{14}$ Ante la persistencia de la fuga, es necesario tener alternativas quirúrgicas, que deberán incluir la gastrectomía total con alguna forma de restitución del tránsito digestivo. Recientemente, se describió una nueva forma de drenaje con catéter 18 fr por gastrostomía endoscópica colocado sobre guía de alambre con técnica de jalón (pull) más catéter peritoneal asistido por laparoscopia tipo cola de cochino (pigtail) y yeyunostomía, con buenos resultados; todo este procedimiento se ha llamado "Técnica de Rendezvous". ${ }^{15}$

En 2015 se describió el uso de megaclips sobre el endoscopio llamados "trampas para oso", conocidos también como "Ovesco", aunado al empleo de megaférulas coreanas recubiertas, con resultados prometedores. ${ }^{16}$ 
Aunque la tomografía axial computada es un buen método para diagnosticar fuga en la MGL, aún tiene una falla hasta del $33 \%$, por lo que ante la sospecha clínica, lo mejor es intervenir por vía laparoscópica en forma temprana, con dos importantes ventajas: diagnóstico certero de la fuga y tratamiento quirúrgico. ${ }^{17-19}$ Deberán descartarse como causas de obstrucción a nivel de la incisura angularis la torsión axial o sutura imbricante muy estrecha. Otra forma de tratamiento de fístulas crónicas es la aplicación de asa en Y de Roux a nivel de la fístula para derivación interna, cuando han fallado los métodos antes descritos. ${ }^{20,21}$ Si no se cuenta con los medios o experiencia suficiente, es necesario asesorarse o trasladar al paciente a otro nivel de atención médica.

\section{REFERENCIAS}

1. Chu CA, Gagner M, Quinn T, et al. Two-stage laparoscopic biliopancreatic diversion with duodenal switch: an alternative approach to super-super morbid obesity (abstract). Surg Endosc. 2002; 16: S069.

2. Eid GM, Brethauer S, Mattar SG, Titcher RL, Gourash W, Schauer PR. Laparoscopic sleeve gastrectomy for super obese patients: forty-eigth percent excess weigth loss after 6 to 8 years with 93\% follow-up. Ann Surg. 2012; 256: 262-265.

3. Yehoshua RT, Eidelman LA, Stein M, Fichman S, Mazor A, Chen J, et al. Laparoscopic sleeve gastrectomy, volume and pressure assessment. Obes Surg. 2008; 18: 1083-1038.

4. Menenakos E, Konstantinos MS, Albanopoulos K, Papailiou J, Teodorou D, Leandros E. Laparoscopic sleeve gastrectomy performed with intent to treat morbid obesity: a prospective single-center study of 261 patients with a median follow up of 1 year. Obes Surg. 2010; 20: 276-282.

5. Burgos AM, Braghetto I, Cscendes A, Maluenda F, Korn $\mathrm{O}$, Yarmuch J, et al. Gastric leak after laparoscopic-sleeve gastrectomy for obesity. Obes Surg. 2009; 19: 1672-1677.

6. Serra C, Baltasar A, Andero L, Pérez N, Bou R, Bengochea $M$, et al. Treatment of gastric leaks with self expanding stents after sleeve gastrectomy. Obes Surg. 2007; 17: 866-872.

7. Kauer WK, Stein HJ, Dittler HJ, Siewert JR. Stent implantation as a treatment option in patients with thoracic anastomotic leaks after esophagectomy. Surg Endosc. 2008; 22: 50-53.

8. Sporn E, Miedema BW, Astudillo JA, Thaler K. Lessons learned establishing an animal model for endoscopic stent placement to treat gastroyeyunal anastomotic leaks after gastric bypass. Obes Surg. 2009; 19: 1163-1169.

9. Lindenmann J, Matzi V, Porubsky C, Anneg U, Sankin $\mathrm{O}$, Gabor S, et al. Self-expandable covered metal tra- cheal type stent for sealing cervical anastomotic leak after esophagectomy and gastric pull-up: pitfalls and posibilities. Ann Thorac Surg. 2008; 85: 354-356.

10. Ramos AC, Ramos MG, Campos JM, Galvao Neto M dos P, Bastos EL. Laparoscopic total gastrectomy as an alternative treatment to postsleeve chronic fistula. Surg Obes Relat Dis. 2015; 11: 552-556.

11. Huerta S, DeShields S, Shiner R, Li Z, Liu C, Sawicki M, et al. Safety and efficacy of postoperative continuous positive airway pressure to prevent pulmonary complications after Roux-en-Y gastric bypass. J Gastrointest Surg. 2002; 6: 354-358.

12. Youssef T, Abdalla E, El-Alfly K, Dawoud I, Morshed $M$, Farid M. Impact of botulinium neurotoxin pyloric injection during laparoscopic sleeve gastrectomy on postoperative gastric leak: a clinical randomized study. Obes Surg. 2016; 26: 494-504.

13. Braghetto I, Davanzo C, Korn O, Csendes A, Valladares $H$, Herrera $E$, et al. Scintigraphic evaluation of gastric emptying on obese patients submitted to sleeve gastrectomy compared to normal subjects. Obes Surg. 2009; 19: 1515-1521.

14. Tan JT, Kariyawasam S, Wijeratne T, Chandraratna HS. Diagnosis and management of gastric leaks after laparoscopic sleeve gastrectomy for morbid obesity. Obes Surg. 2010; 20: 403-440.

15. Soufron J. Leak or fistula after sleeve gastrectomy, treatment with Pigtail drain by the Rendezvous Technique. Obes Surg. 2015; 25: 1979-1980.

16. Shehab HM, Hakky SM, Gawdat KA. An endoscopic strategy combining mega stents and over the scope clips for the management of postbariatric surgery leaks and fistulas (with video). Obes Surg. 2016; 26: 941-948.

17. Arteaga-González I, Martín-Malagón A, Martin-Pérez J, Carrillo-Pallarés A. Ulsefulness of clinical signs and diagnostic test for suspected leaks in bariatric surgery. Obes Surg. 2015; 25: 1680-1684.

18. ASMBS guideline on the prevention and detection of gastrointestinal leak after gastric bypass including the role of imaging and surgical exploration. Surg Obes Relat Dis. 2009; 5: 293-296.

19. Hussain A, El-Hasani S. Diagnostic laparoscopy or CT Scan to diagnose the leak following Bariatric Surgery. Obes Surg. 2016; 26: 617-618.

20. Van de Vrande S, Himpens J, El Mourad H, Debaerdemaeker R, Leman G. Management of chronic proximal fistulas after sleeve gastrectomy by laparoscopic Rouxlimb placement. Surg Obes Relat Dis. 2013; 9: 856-861.

21. Nedelcu M, Skalli M, Deneve E, Fabre J, Nocca D. Surgical management of chronic fistula after sleeve gastrectomy. Surg Obes Relat Dis. 2013; 9: 879-884.

Correspondencia:

Dr. César Romero Mejía

J. Clemente Orozco Núm. 1407,

Zona Río, Tijuana, Baja California, 22320,

México.

Tel: (664) 6343301 y 6343302

E-mail: romerocirugia@hotmail.com 